\title{
Bijective proofs for Schur function identities which imply Dodgson's condensation formula and Plücker relations
}

\author{
Markus Fulmek \\ Institut für Mathematik der Universität Wien \\ Strudlhofgasse 4, A-1090 Wien, Austria \\ Markus.Fulmek@Univie.Ac.At \\ Michael Kleber \\ Massachusetts Institute of Technology \\ 77 Massachusetts Avenue, Cambridge, MA 02139, USA \\ Kleber@Math.Mit.Edu
}

Submitted: July 3, 2000; Accepted: March 7, 2001.

MR Subject Classifications: 05E05 05E15

\begin{abstract}
We present a "method" for bijective proofs for determinant identities, which is based on translating determinants to Schur functions by the Jacobi-Trudi identity. We illustrate this "method" by generalizing a bijective construction (which was first used by Goulden) to a class of Schur function identities, from which we shall obtain bijective proofs for Dodgson's condensation formula, Plücker relations and a recent identity of the second author.
\end{abstract}

\section{Introduction}

Usually, bijective proofs of determinant identities involve the following steps (cf., e.g, [19, Chapter 4] or [23, 24]):

- Expansion of the determinant as sum over the symmetric group,

- Interpretation of this sum as the generating function of some set of combinatorial objects which are equipped with some signed weight,

- Construction of an explicit weight- and sign-preserving bijection between the respective combinatorial objects, maybe supported by the construction of a signreversing involution for certain objects. 
Here, we will present another "method" of bijective proofs for determinant identitities, which involves the following steps:

- First, we replace the entries $a_{i, j}$ of the determinants by $h_{\lambda_{i}-i+j}$ (where $h_{m}$ denotes the $m$-th complete homogeneous function),

- Second, by the Jacobi-Trudi identity we transform the original determinant identity into an equivalent identity for Schur functions,

- Third, we obtain a bijective proof for this equivalent identity by using the interpretation of Schur functions in terms of nonintersecting lattice paths. (In this paper, we shall achieve this with a construction which was used for the proof of a Schur function identity [3, Theorem 1.1] conjectured by Ciucu.)

We show how this method applies naturally to provide elegant bijective proofs of Dodgson's Condensation Rule [2] and the Plücker relations.

The bijective construction we use here was (to the best of our knowledge) first used by I. Goulden [7]. (The first author is grateful to A. Hamel [8] for drawing his attention to Goulden's work.) Goulden's exposition, however, left open a small gap, which we shall close here.

The paper is organized as follows: In Section 2, we present the theorems we want to prove, and explain Steps 1 and 2 of our above "method" in greater detail. In Section 3, we briefly recall the combinatorial definition of Schur functions and the Gessel-Viennotapproach. In Section 4, we explain the bijective construction employed in Step 3 of our "method" by using the proof of a Theorem from Section 2 as an illustrating example. There, we shall also close the small gap in Goulden's work. In Section 5, we "extract" the general structure underlying the bijection: As it turns out, this is just a simple graphtheoretic statement. From this we may easily derive a general "class" of Schur function identities which follow from these considerations. In order to show that these quite general identitities specialize to something useful, we shall deduce the Plücker relations, using again our "method". In Section 7, we turn to a theorem [11, Theorem 3.2] recently proved by the second author by using Plücker relations: We explain how this theorem fits into our construction and give a bijective proof using inclusion-exclusion.

\section{Exposition of identities and proofs}

The origin of this paper was the attempt to give a bijective proof of the following identity for Schur functions, which arose in work of Kirillov [10]:

Theorem 1 Let $c, r$ be positive integers; denote by $\left[c^{r}\right]$ the partition consisting of $r$ rows with constant length $c$. Then we have the following identity for Schur functions:

$$
\left(s_{\left[c^{r}\right]}\right)^{2}=s_{\left[c^{r-1}\right]} \cdot s_{\left[c^{r+1}\right]}+s_{\left[(c-1)^{r}\right]} \cdot s_{\left[(c+1)^{r}\right]} .
$$


(See [18, 7.10], [5], [13] or [16] for background information on Schur functions; in order to keep our exposition self-contained, a combinatorial definition is given in Section 4.)

The identity (1) was recently considered by the second author [11, Theorem 4.2], who also gave a bijective proof, and generalized it considerably [11, Theorem 3.2].

The construction we use here does in fact prove a more general statement:

Theorem 2 Let $\left(\lambda_{1}, \lambda_{2}, \ldots, \lambda_{r+1}\right)$ be a partition, where $r>0$ is some integer. Then we have the following identity for Schur functions:

$$
s_{\left(\lambda_{1}, \ldots, \lambda_{r}\right)} \cdot s_{\left(\lambda_{2}, \ldots, \lambda_{r+1}\right)}=s_{\left(\lambda_{2}, \ldots, \lambda_{r}\right)} \cdot s_{\left(\lambda_{1}, \ldots, \lambda_{r+1}\right)}+s_{\left(\lambda_{2}-1, \ldots, \lambda_{r+1}-1\right)} \cdot s_{\left(\lambda_{1}+1, \ldots, \lambda_{r}+1\right)} .
$$

Clearly, Theorem 1 is a direct consequence of Theorem 2: Simply set $\lambda_{1}=\cdots=$ $\lambda_{r+1}=c$.

Theorem 2, however, is in fact equivalent to Dodgson's condensation formula [2], which is also known as Desnanot-Jacobi's adjoint matrix theorem (see [1, Theorem 3.12]: According to [1], Lagrange discovered this theorem for $n=3$, Desnanot proved it for $n \leq 6$ and Jacobi published the general theorem [9], see also [14, vol. I, pp. 142]):

Theorem 3 Let $A$ be an arbitrary $(r+1) \times(r+1)$-determinant. Denote by $A_{\left\{r_{1}, r_{2}\right\},\left\{c_{1}, c_{2}\right\}}$ the minor consisting of rows $r_{1}, r_{1}+1, \ldots, r_{2}$ and columns $c_{1}, c_{1}+1, \ldots, c_{2}$ of $A$. Then we have the following identity:

$$
A_{\{1, r+1\},\{1, r+1\}} A_{\{2, r\},\{2, r\}}=A_{\{1, r\},\{1, r\}} A_{\{2, r+1\},\{2, r+1\}}-A_{\{2, r+1\},\{1, r\}} A_{\{1, r\},\{2, r+1\}} .
$$

The transition from Theorem 3 to Theorem 2 is established by the Jacobi-Trudi identity (see $[13, \mathrm{I},(3.4)]$ ), which states that for any partition $\lambda=\left(\lambda_{1}, \ldots, \lambda_{r}\right)$ of length $r$ we have

$$
s_{\lambda}=\operatorname{det}\left(h_{\lambda_{i}-i+j}\right)_{i, j=1}^{r},
$$

where $h_{m}$ denotes the $m$-th complete homogeneous symmetric function: Setting $A_{i, j}:=$ $h_{\lambda_{i}-i+j}$ for $1 \leq i, j \leq r+1$ in Theorem 3 and using identity (4) immediately yields (2).

That the seemingly weaker statement of Theorem 2 does in fact imply Theorem 3 is due to the following observation: Choose $\lambda$ so that the numbers $\lambda_{i}-i+j$ are all distinct for $1 \leq i, j \leq(r+1)$ (e.g., $\lambda=\left((r+1) r, r^{2},(r-1) r, \ldots, r\right)$ would suffice) and rewrite (2) as a determinantal expression according to the Jacobi-Trudi identity (4). This yields a special case of identity (3) with $A_{i, j}:=h_{\lambda_{i}-i+j}$ as above. Now recall that the complete homogeneous symmetric functions are algebraically independent (see, e.g., [21]), whence the identity (3) is true for generic $A_{i, j}$. For later use, we record this simple observation in a more general fashion:

Observation 4 Let $\mathcal{I}$ be an identity involving determinants of homogeneous symmetric functions $h_{n}$, where $n$ is some nonnegative integer. Then $\mathcal{I}$ is, in fact, equivalent to a general determinant identity which is obtained from $\mathcal{I}$ by considering each $h_{n}$ as a formal variable. 
So far, the promised proof (to be given in Section 4) of Theorem 2 would give a new bijective proof of Dodgson's Determinant-Evaluation Rule (a beautiful bijective proof was also given by Zeilberger [23]). But we can do a little better: Our bijective construction does, in fact, apply to a quite general "class of Schur function identities", a special case of which implies the Plücker relations (also known as Grassmann-Plücker syzygies), see, e.g., [21], or [22, Chapter 3, Section 9, formula II]:

Theorem 5 (Plücker relations) Consider an arbitrary $2 n \times n$-matrix with row indices $1,2, \ldots, 2 n$. Denote the $n \times n$-minor of this matrix consisting of rows $i_{1}, \ldots, i_{n}$ by $\left[i_{1}, \ldots, i_{n}\right]$.

Consider some fixed list of integers $1 \leq r_{1}<r_{2}<\cdots<r_{k} \leq n, 0 \leq k \leq n$. Then we have:

$$
\begin{aligned}
& {[1,2, \ldots, n] \cdot[n+1, n+2, \ldots, 2 n]=} \\
& \sum_{n+1 \leq t_{1}<t_{2}<\cdots<t_{k} \leq 2 n}\left[1, \ldots, t_{1}, \ldots, t_{k}, \ldots, n\right] \cdot\left[n+1, \ldots, r_{1}, \ldots, r_{k}, \ldots, 2 n\right],
\end{aligned}
$$

where the notation of the summands means that rows $r_{i}$ were exchanged with rows $t_{i}$, respectively.

This is achieved by observing that (5) can be specialized to a Schur function identity of the form

$$
s_{\lambda} s_{\mu}=\sum_{\lambda^{\prime}, \mu^{\prime}} s_{\lambda^{\prime}} s_{\mu^{\prime}}
$$

where $\lambda$ and $\mu$ are partitions with the same number $n$ of parts, and where the sum is over certain pairs $\lambda^{\prime}, \mu^{\prime}$ derived from $\lambda, \mu$ (to be described later). This Schur function identity belongs to the "class of identities" which follow from the bijective construction. By applying Observation 4 with suitable $\lambda$ and $\mu$, we may deduce (5).

Remark 6 Summing equation (5) over all possible choices of subsets $\left\{r_{1}, \ldots, r_{k}\right\}$ yields the determinant identity behind Ciucu's Schur function identity [3, Theorem 1.1]

$$
\sum_{A \subset T:|A|=k} s_{\lambda(A)} s_{\lambda(T-A)}=2^{k} s_{\lambda\left(t_{2}, \ldots, t_{2 k}\right)} s_{\lambda\left(t_{1}, \ldots, t_{2 k-1}\right)},
$$

where $T=\left\{t_{1}<\cdots<t_{2 k}\right\}$ is some set of positive integers and $\lambda\left(\left\{t_{i_{1}}<\cdots<t_{i_{r}}\right\}\right)$ denotes the partition with parts $t_{i_{r}}-r+1 \geq \cdots \geq t_{i_{2}}-1 \geq t_{i_{1}}$.

Remark 7 The Plücker relations (5) appear in a slightly different notation as Theorem 2 in [15], together with another elegant proof.

Moreover, the bijective method yields a proof of the second author's theorem [11, Theorem 3.2]: Since this theorem is rather complicated to state, we defer it to Section 7. 


\section{Combinatorial background and definitions}

As usual, an $r$-tuple $\lambda=\left(\lambda_{1}, \lambda_{2}, \ldots, \lambda_{r}\right)$ with $\lambda_{1} \geq \lambda_{2} \geq \cdots \geq \lambda_{r} \geq 0$ is called a partition of length $r$. The Ferrers board $F(\lambda)$ of $\lambda$ is an array of cells with $r$ left-justified rows and $\lambda_{i}$ cells in row $i$.

An $N$-semistandard Young tableau of shape $\lambda$ is a filling of the cells of $F(\lambda)$ with integers from the set $\{1,2, \ldots, N\}$, such that the numbers filled into the cells weakly increase in rows and strictly increase in columns (see the right picture of Figure 1 for an illustration).

Schur functions, which are irreducible general linear characters, can be combinatorially defined by means of $N$-semistandard Young tableaux (see [13, I, (5.12)], [16, Def. 4.4.1], [17, Def. 5.1]):

$$
s_{\lambda}\left(x_{1}, x_{2}, x_{3}, \ldots, x_{N}\right)=\sum_{\mathbf{T}} w(\mathbf{T}),
$$

where the sum is over all $N$-semistandard Young tableaux $\mathbf{T}$ of shape $\lambda$. Let $m(\mathbf{T}, k)$ be the number of entries $k$ in the tableau $\mathbf{T}$. The weight $w(\mathbf{T})$ of $\mathbf{T}$ is defined as follows:

$$
w(\mathbf{T})=\prod_{k=1}^{N} x_{k}^{m(\mathbf{T}, k)} .
$$

The Gessel-Viennot interpretation [6] of semistandard Young tableaux of shape $\lambda$ as nonintersecting lattice paths (see the left picture of Figure 1 for an illustration) allows an equivalent definition of Schur functions:

$$
s_{\lambda}\left(x_{1}, x_{2}, x_{3}, \ldots, x_{N}\right)=\sum_{\mathbf{P}} w(\mathbf{P}),
$$

where the sum is over all $r$-tuples $\mathbf{P}=\left(P_{1}, P_{2}, \ldots, P_{r}\right)$ of lattice paths (in the integer lattice, i.e., the directed graph with vertices $\mathbb{Z} \times \mathbb{Z}$ and $\operatorname{arcs}$ from $(j, k)$ to $(j+1, k)$ and from $(j, k)$ to $(j, k+1)$ for all $j, k)$, where $P_{i}$ starts at $(-i, 1)$ and ends at $\left(\lambda_{i}-i, N\right)$, and where no two paths $P_{i}$ and $P_{j}$ have a lattice point in common (such an $r$-tuple is called nonintersecting).

The weight $w(\mathbf{P})$ of an $r$-tuple $\mathbf{P}=\left(P_{1}, P_{2}, \ldots, P_{r}\right)$ of paths is defined by:

$$
w(\mathbf{P})=\prod_{i=1}^{r} w\left(P_{i}\right)
$$

The weight $w(P)$ of a single path $P$ is defined as follows: Let $n(P, k)$ be the number of horizontal steps at height $k$ (i.e., directed arcs from some $(j, k)$ to $(j+1, k))$ that belong to path $P$, then we define

$$
w(P)=\prod_{k=1}^{N} x_{k}^{n(P, k)}
$$


Figure 1: Illustration of a 6-semistandard Young tableau and its associated lattice paths for $\lambda=(4,3,2)$.

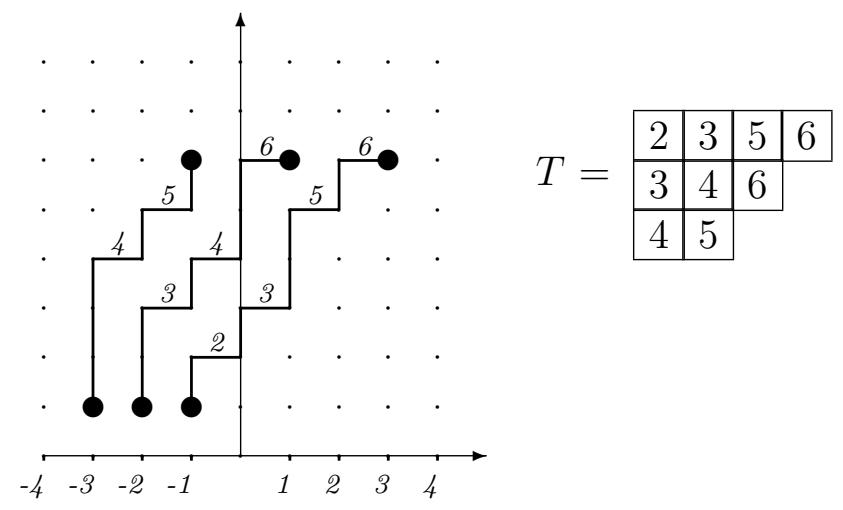

Figure 2: Illustration of a 6 -semistandard skew Young tableau and its associated lattice paths for $\lambda=(4,3,2)$ and $\mu=(1,0,0)$.

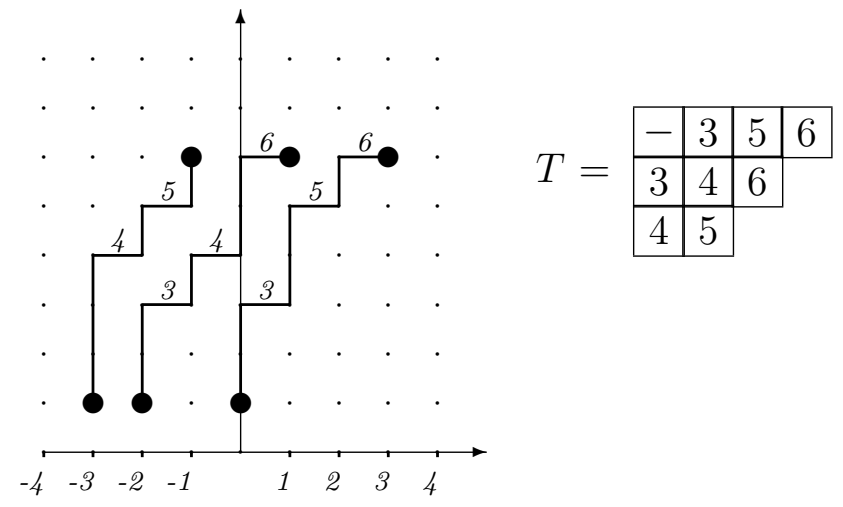

That these definitions are in fact equivalent is due to a weight-preserving bijection between tableaux and nonintersecting lattice paths. The Gessel-Viennot method [6] builds on the lattice path definition to give a bijective proof of the Jacobi-Trudi identity (4) (see, e.g., [16, ch. 4], [20] or [4]).

Next, we give a combinatorial definition for skew Schur functions: Let $\lambda=\left(\lambda_{1}, \ldots, \lambda_{r}\right)$ and $\mu=\left(\mu_{1}, \ldots, \mu_{r}\right)$ be partitions with $\mu_{i} \leq \lambda_{i}$ for $1 \leq i \leq r$; here, we allow $\mu_{i}=0$.

The skew Ferrers board $F(\lambda / \mu)$ of $(\lambda, \mu)$ is an array of cells with $r$ left-justified rows and $\lambda_{i}-\mu_{i}$ cells in row $i$, where the first $\mu_{i}$ cells in row $i$ are missing.

An $N$-semistandard skew Young tableau of shape $\lambda / \mu$ is a filling of the cells of $F(\lambda / \mu)$ with integers from the set $\{1,2, \ldots, N\}$, such that the numbers filled into the cells weakly increase in rows and strictly increase in columns (see the right picture of Figure 2 for an illustration). 
Then we have the following definition for skew Schur functions:

$$
s_{\lambda / \mu}\left(x_{1}, x_{2}, x_{3}, \ldots, x_{N}\right)=\sum_{\mathbf{T}} w(\mathbf{T}),
$$

where the sum is over all $N$-semistandard skew Young tableaux $\mathbf{T}$ of shape $\lambda / \mu$, where the weight $w(\mathbf{T})$ of $\mathbf{T}$ is defined as before.

Equivalently, we may define:

$$
s_{\lambda / \mu}\left(x_{1}, x_{2}, x_{3}, \ldots, x_{N}\right)=\sum_{\mathbf{P}} w(\mathbf{P})
$$

where the sum is over all $r$-tuples $\mathbf{P}=\left(P_{1}, P_{2}, \ldots, P_{r}\right)$ of nonintersecting lattice paths, where $P_{i}$ starts at $\left(\mu_{i}-i, 1\right)$ and ends at $\left(\lambda_{i}-i, N\right)$ (see the left picture of Figure 2 for an illustration), and where the weight $w(\mathbf{P})$ of such an $r$-tuple $\mathbf{P}$ is defined as before.

\section{Bijective proof of Theorem 2}

Proof: Let us start with a combinatorial description for the objects involved in (2): By the Gessel-Viennot interpretation of Schur functions as generating functions of nonintersecting lattice paths, we may view the left-hand side of the equation as the weight of all pairs $\left(\mathbf{P}^{g}, \mathbf{P}^{b}\right)$, where $\mathbf{P}^{g}$ and $\mathbf{P}^{b}$ are $r$-tuples of nonintersecting lattice paths. The paths of $\mathbf{P}^{g}$ are coloured green, the paths of $\mathbf{P}^{b}$ are coloured blue. The $i$-th green path $P_{i}^{g}$ starts at $(-i, 1)$ and ends in $\left(\lambda_{i}-i, N\right)$. The $i$-th blue path $P_{i}^{b}$ starts at $(-i-1,1)$ and ends in $\left(\lambda_{i+1}-i-1, N\right)$. For an illustration, see the upper left pictures in Figures 3 and 4 , where green paths are drawn with full lines and blue paths are drawn with dotted lines.

For the right-hand side of (2), we use the same interpretation. We may view the first term as the weight of all pairs $\left(\mathbf{A}^{g}, \mathbf{A}^{b}\right)$, where $\mathbf{A}^{g}$ is an $(r-1)$-tuple of nonintersecting lattice paths and $\mathbf{A}^{b}$ is an $(r+1)$-tuple of nonintersecting lattice paths. The paths of $\mathbf{A}^{g}$ are coloured green, the paths of $\mathbf{A}^{b}$ are coloured blue. The $i$-th green path $A_{i}^{g}$ starts at $(-i-1,1)$ and ends in $\left(\lambda_{i+1}-i-1, N\right)$. The $i$-th blue path $A_{i}^{b}$ starts at $(-i, 1)$ and ends in $\left(\lambda_{i}-i, N\right)$. For an illustration, see the upper right picture in Figure 3.

In the same way, we may view the second term as the weight of all pairs $\left(\mathbf{B}^{g}, \mathbf{B}^{b}\right)$, where $\mathbf{B}^{g}$ and $\mathbf{B}^{b}$ are $r$-tuples of nonintersecting lattice paths. The paths of $\mathbf{B}^{g}$ are coloured green, the paths of $\mathbf{B}^{b}$ are coloured blue. The $i$-th green path $B_{i}^{g}$ starts at $(-i, 1)$ and ends in $\left(\lambda_{i+1}-i-1, N\right)$. The $i$-th blue path $B_{i}^{b}$ starts at $(-i-1,1)$ and ends in $\left(\lambda_{i}-i, N\right)$. For an illustration, see the upper right picture in Figure 4.

In any case, the weight of some pair of paths $(\mathbf{P}, \mathbf{Q})$ is defined as follows:

$$
w(\mathbf{P}, \mathbf{Q}):=w(\mathbf{P}) \cdot w(\mathbf{Q}) .
$$

What we want to do is to give a weight-preserving bijection between the objects on the left side and on the right side:

$$
\left\{\left(\mathbf{P}^{g}, \mathbf{P}^{b}\right)\right\} \leftrightarrow\left(\left\{\left(\mathbf{A}^{g}, \mathbf{A}^{b}\right)\right\} \cup\left\{\left(\mathbf{B}^{g}, \mathbf{B}^{b}\right)\right\}\right)
$$


Figure 3: Illustration of the construction in the proof, case A: $r=3,\left(\lambda_{1}, \lambda_{2}, \lambda_{3}, \lambda_{4}\right)=$ $(5,4,3,2)$.

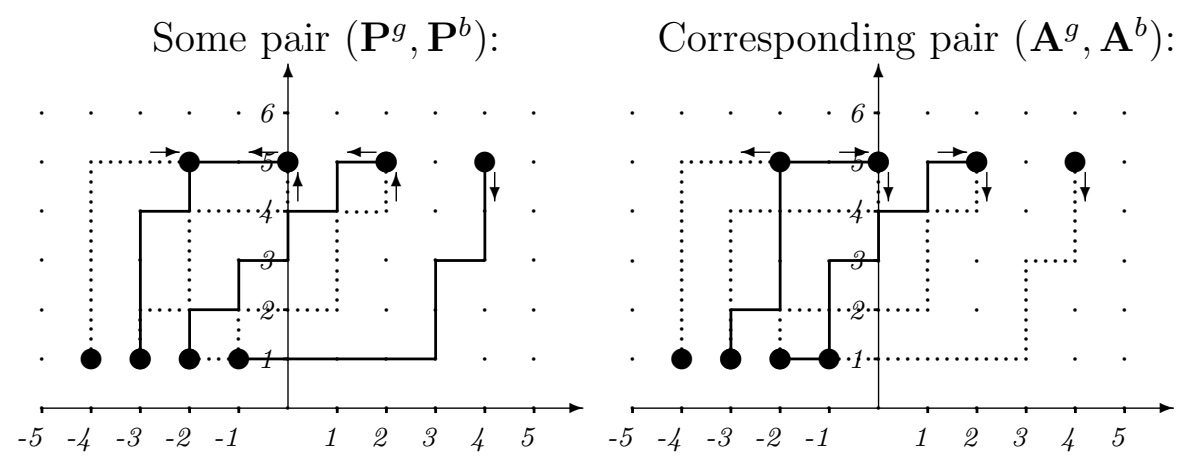

The changing trail starting in $(4,5)$ :

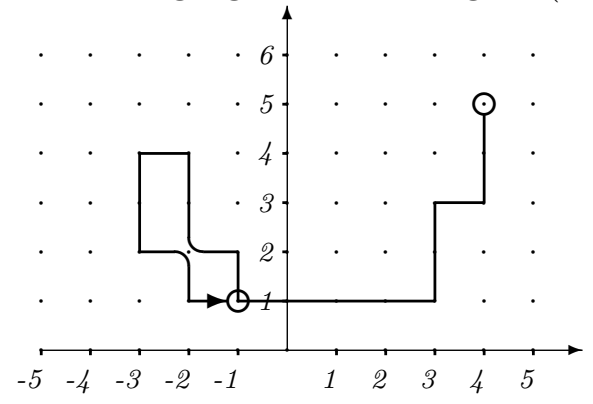


Figure 4: Illustration of the construction in the proof, case B: $r=3,\left(\lambda_{1}, \lambda_{2}, \lambda_{3}, \lambda_{4}\right)=$ $(5,4,3,2)$.

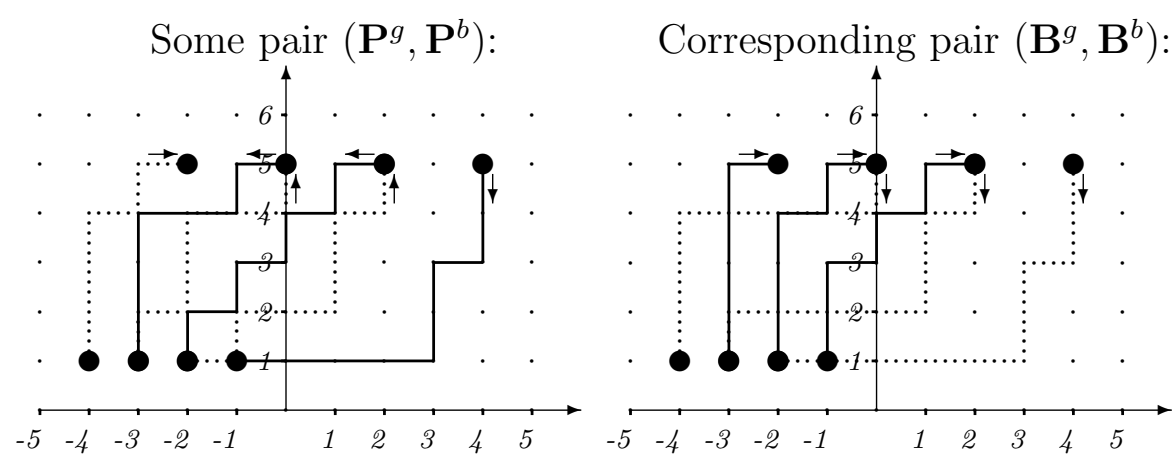

The changing trail starting in $(4,5)$ :

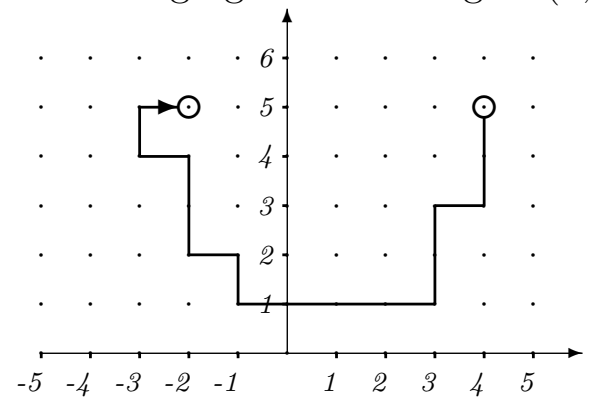


Clearly, such a bijection would establish (2).

The basic idea is very simple and was already used in [7] and in [3]: Since it will be reused later, we state it here quite generally:

Definition 8 Let $\mathbf{P}^{1}, \mathbf{P}^{2}$ be two arbitrary families of nonintersecting lattice paths. The paths $P_{i}^{1}$ of the first family are coloured with colour blue, the paths $P_{j}^{2}$ of the second family are coloured with colour green.

Let $G\left(\mathbf{P}^{1}, \mathbf{P}^{2}\right)$ be the "two-coloured" graph made up by $\mathbf{P}^{1}$ and $\mathbf{P}^{2}$ in the obvious sense. Observe that there are the two possible orientations for any edge in that graph: When traversing some path, we may either move "right-upwards" (this is the "original" orientation of the paths) or "left-downwards".

A changing trail is a trail in $G\left(\mathbf{P}^{1}, \mathbf{P}^{2}\right)$ with the following properties:

- Subsequent edges of the same colour are traversed in the same orientation, subsequent edges of the opposite colour are traversed in the opposite orientation.

- At every intersection of green and blue paths, colour and orientation are changed if this is possible (i.e., if there is an adjacent edge of opposite colour and opposite orientation); otherwise the trail must stop there.

- The trail is maximal in the sense that it cannot be extended by adjoining edges (in a way which is consistent with the above conditions) at its start or end.

Note that for every edge e, there is a unique changing trail which contains e: E.g., consider some blue edge which is right-or upwards-directed and enters vertex $v$. If there is an intersection at $v$, and if there is a green edge leaving $v$ (in opposite direction left or downwards), then the trail must continue with this edge; otherwise it must stop at $v$. If there is no intersection at $v$, and if there is a blue edge leaving $v$ (in the same direction right or upwards), then the trail must continue with this edge; otherwise it must stop at $v$.

Note that a changing trail is either "path-like", i.e., has obvious starting point and end point (clearly, these must be the end points or starting points of some path from either $\mathbf{P}^{1}$ or $\left.\mathbf{P}^{2}\right)$, or it is "cycle-like", i.e., is a closed trail.

Let us return from general definitions to our concrete case: Starting with an object $\left(\mathbf{P}^{g}, \mathbf{P}^{b}\right)$ from the left-hand side of $(7)$, we interpret this pair of lattice paths as a graph $G\left(\mathbf{P}^{g}, \mathbf{P}^{b}\right)$ with green and blue edges. (See the upper left pictures in Figures 3 and 4 .)

Next, we determine the changing trail which starts at the rightmost endpoint $\left(\lambda_{1}-\right.$ $1, N)$ : Follow the green edges downward or to the left; at every intersection, change colour and orientation, if this is possible; otherwise stop there. Clearly, this changing trail is "path-like". (See Figures 3 and 4 for an illustration: There, the orientation of edges is indicated by small arrows in the upper pictures; the lower pictures show the corresponding changing trails.)

Now we change colours green to blue and vice versa along this changing trail: It is easy to see that this recolouring yields nonintersecting tuples of green and blue lattice paths. 
Note that there are exactly two possible cases:

Case A: The changing trail stops at the rightmost starting point, $(-1,1)$, of the lattice paths. In this case, from the recolouring procedure we obtain an object $\left(\mathbf{A}^{g}, \mathbf{A}^{b}\right)$; see the upper right picture in Figure 3.

Case B: The changing trail stops at the the leftmost endpoint, $\left(\lambda_{r+1}-r-1, N\right)$, of the lattice paths. In this case, from the recolouring procedure we obtain an object $\left(\mathbf{B}^{g}, \mathbf{B}^{b}\right)$; see the upper right picture in Figure 4.

It is clear that altogether this gives a mapping of the set of all objects $\left(\mathbf{P}^{g}, \mathbf{P}^{b}\right)$ into the union of the two sets of all objects $\left(\mathbf{A}^{g}, \mathbf{A}^{b}\right)$ and $\left(\mathbf{B}^{g}, \mathbf{B}^{b}\right)$, respectively. Of course, this mapping is weight-preserving. It is also injective since the above construction is reversed by simply repeating it, i.e, determine the changing trail starting at the rightmost endpoint $\left(\lambda_{1}-1, N\right)$ (this trail is exactly the same as before, only the colours are exchanged) and change colours. For an illustration, read Figures 3 and 4 from right to left.

So what is left to prove is surjectivity: To this end, it suffices to prove that if we apply our (injective) recolouring construction to an arbitrary object $\left(\mathbf{A}^{g}, \mathbf{A}^{b}\right)$ or $\left(\mathbf{B}^{g}, \mathbf{B}^{b}\right)$, we do always get an object $\left(\mathbf{P}^{g}, \mathbf{P}^{b}\right)$; i.e., two $\mathrm{r}$-tuples of nonintersecting lattice paths, coloured green and blue, and with the appropriate starting points and endpoints.

We do have something to prove: Note that in both cases, A (see Figure 3) and B (see Figure 4), there is prima vista a second possible endpoint for the changing trail, namely the leftmost starting point, $(-r-1,1)$, of the lattice paths, where the leftmost blue path starts. If this endpoint could actually be reached, then the resulting object would clearly not be of type $\left(\mathbf{P}^{g}, \mathbf{P}^{b}\right)$. So we have to show that this is impossible. (Goulden left out this indispensable step in [7, Theorem 2.2], but we shall close this small gap immediately.)

Observation 9 The following properties of changing trails are immediate:

- If some edge of a changing trail is used by paths of both colours green and blue, then it is necessarily traversed in both orientations and thus forms a changing trail (which is "cycle-like") by itself.

- Two changing trails may well touch each other (i.e., have some vertex in common), but can never cross.

Now observe that in Case A, there is also a second possible starting point of a "pathlike" changing trail, namely the left-most endpoint $\left(\lambda_{r+1}-r-1, N\right)$ of the lattice paths (see the left picture in Figure 5). Likewise, in Case B, there is a second possible starting point of a "path-like" changing trail, namely the rightmost starting point $(-1,1)$ of the lattice paths (see the right picture in Figure 5).

In both cases, if the changing trail starting in $\left(\lambda_{1}-1, N\right)$ would reach the leftmost starting point $(-r-1,1)$ of the lattice paths, it clearly would cross this other "path-like" changing trail; a contradiction to Observation 9. (The pictures in Figure 5 shows these other changing trails for the examples in Figures 3 and 4, respectively.) 
Figure 5: Illustration of the second changing trails for cases A and B.

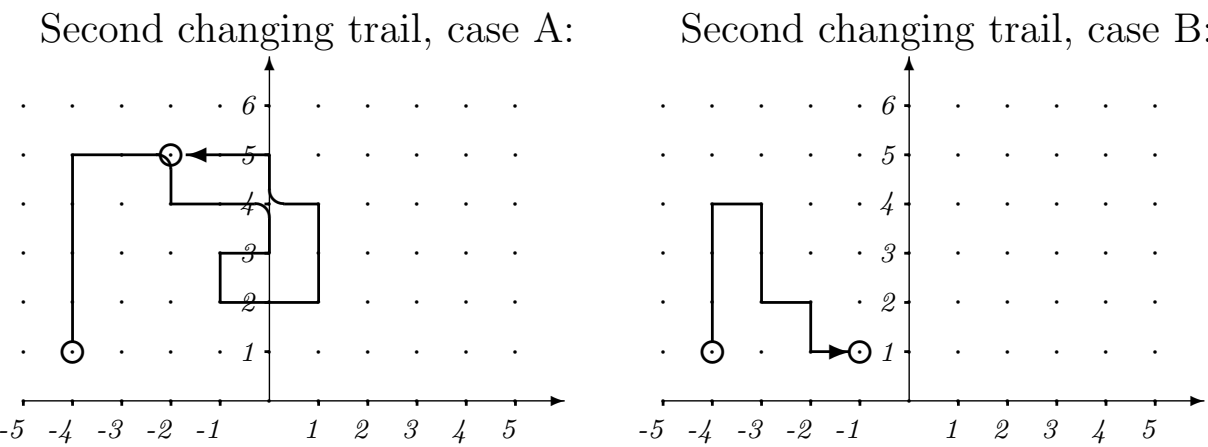

\section{The bijective construction, generalized}

It is immediately obvious that the bijective construction used in the proof of Theorem 2 is not at all restricted to the special situation of Theorem 2: We can always consider the product of two (arbitrary) skew Schur functions as generating functions of certain "twocoloured graphs" derived from the lattice path interpretation, as above. Determining the changing trails which start in some fixed set of starting points and recolouring their edges will always yield an injective (and, clearly, weight-preserving) mapping: The only issue which needs extra care is surjectivity.

In the proof of Theorem 2 we saw that the argument showing surjectivity boils down to a very simple graph-theoretic reasoning. We shall recast this simple reasoning into a general statement:

Observation 10 Consider the complete graph $K_{2 n}$ with $2 n$ vertices, numbered $1, \ldots, 2 n$, and represent its vertices as points on the unit circle (i.e., vertex number $m$ is represented as $\left.e^{2 m \pi \sqrt{-1}}\right)$; represent the edges as straight lines connecting the corresponding vertices. Call a matching in this graph noncrossing if no two of its edges cross each other in this geometric representation (see Figure 6 for an illustration). Then we have:

Any edge which belongs to a perfect noncrossing matching must connect an oddnumbered vertex to an even-numbered vertex.

Remark 11 Note that the number of perfect noncrossing matchings in $K_{2 n}$ is the Catalan number $C_{n}$ (see [18, p. 222]).

Remark 12 Note that the argument proving surjectivity in Theorem 2 amounts to the fact that the two possible "path-like" changing trails connecting the four possible starting points and end points $\left(\lambda_{1}-1, N\right),\left(\lambda_{r+1}-r-1, N\right),(-r-1,1)$ and $(-1,1)$ must correspond to a noncrossing perfect matching of the complete graph $K_{4}$.

We shall derive a general statement for skew Schur functions:

Let $\lambda=\left(\lambda_{1}, \ldots, \lambda_{r}\right)$ and $\mu=\left(\mu_{1}, \ldots, \mu_{r}\right)$ be partitions with $0 \leq \mu_{i} \leq \lambda_{i}$ for $1 \leq i \leq r$; let $\sigma=\left(\sigma_{1}, \ldots, \sigma_{r}\right)$ and $\tau=\left(\tau_{1}, \ldots, \tau_{r}\right)$ be partitions with $0 \leq \tau_{i} \leq \sigma_{i}$ for $1 \leq i \leq r$. 
Figure 6: Illustration of a perfect noncrossing matching in $K_{8}$.

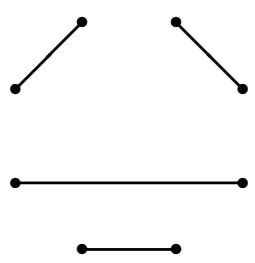

Remark 13 We intentionally allow parts of length 0 in the partitions $\lambda$ and $\sigma$ : This is equivalent to allowing them to have different numbers of parts.

Interpret $s_{\lambda / \mu}$ as the generating function of the family of nonintersecting lattice paths $\left(P_{1}^{b}, \ldots, P_{r}^{b}\right)$, where $P_{i}^{b}$ starts at $\left(\mu_{i}-i, 1\right)$ and ends at $\left(\lambda_{i}-i, N\right)$. Colour the corresponding lattice paths blue.

Interpret $s_{\sigma / \tau}$ as the generating function of the family of nonintersecting lattice paths $\left(P_{1}^{g}, \ldots, P_{r}^{g}\right)$, where $P_{i}^{g}$ starts at $\left(\tau_{i}+t-i, 1\right)$ and ends at $\left(\sigma_{i}+t-i, N\right)$. Colour the corresponding lattice paths green. Here, $t$ is an arbitrary but fixed integer which indicates the horizontal offset of the green paths with respect to the blue paths.

Consider the sequence of possible starting points of "path-like" changing trails of the corresponding two-coloured graph, in the sense of Section 4, where the end-points of the lattice paths appear in order from right to left in this sequence, followed by the starting points of the lattice paths in order from left to right. Note that the number of such points is even, $2 k$, say. More precisely, consider $\left(x_{1}, N\right), \ldots\left(x_{l}, N\right)$, followed by $\left(x_{l+1}, 1\right), \ldots,\left(x_{2 k}, 1\right)$, where

$$
\begin{aligned}
\left\{x_{1}, \ldots, x_{l}\right\}=\left\{i: \lambda_{i}-i \neq \sigma_{j}+t-j \text { for } 1 \leq j \leq r\right\} \cup & \\
& \left\{j: \sigma_{j}+t-j \neq \lambda_{i}-i \text { for } 1 \leq i \leq r\right\},
\end{aligned}
$$

$x_{1}>x_{2}>\cdots>x_{l}$, and where

$$
\begin{aligned}
\left\{x_{l+1}, \ldots, x_{2 k}\right\}=\left\{i: \mu_{i}-i \neq \tau_{j}+t-j \text { for } 1 \leq j \leq r\right\} & \\
& \left\{j: \tau_{j}+t-j \neq \mu_{i}-i \text { for } 1 \leq i \leq r\right\}
\end{aligned}
$$

$x_{l+1}<\cdots<x_{2 k}$.

Denote this sequence of points $\left(x_{i},.\right)$ by $\left(Q_{i}\right), 1 \leq i \leq 2 k$. For $1 \leq i \leq l$, blue points $Q_{i}$ are coloured black and green points $Q_{i}$ are coloured white. For $l+1 \leq i \leq 2 k$, blue points $Q_{i}$ are coloured white and green points $Q_{i}$ are coloured black. Points with even index are called even, points with odd index are called odd. Then the following lemma is immediate:

Lemma 14 A path-like changing trail in the two-coloured graph defined above can only connect points of different colours (out of black and white) and of different parity (by Observation 10); e.g., some white $Q_{2 m}$ and some black $Q_{2 n+1}$. 
Now fix an arbitrary subset of points $\left\{Q_{i_{1}}, \ldots, Q_{i_{m}}\right\}$. Start with an arbitrary twocoloured graph from $s_{\lambda / \mu} s_{\sigma / \tau}$ (interpreted again as the product of the generating functions of the corresponding families of nonintersecting lattice paths) and recolour the changing trails starting in $Q_{i_{1}}, \ldots, Q_{i_{m}}$. In general, this will give another two-coloured graph, which can be interpreted as belonging to some other $s_{\lambda^{\prime} / \mu^{\prime}} s_{\sigma^{\prime} / \tau^{\prime}}$. Take an arbitrary object (i.e., two-coloured graph) from $s_{\lambda^{\prime} / \mu^{\prime}} s_{\sigma^{\prime} / \tau^{\prime}}$ and repeat the same recolouring operation as long as it generates some "new" (yet unseen) object.

The set of objects thus generated decomposes into two disjoint sets: One set, $O_{0}$, encompasses all objects which show the same colouring of points $Q_{i_{1}}, \ldots, Q_{i_{m}}$ as in the starting object; the other, $O_{1}$ encompasses the objects with the opposite colouring for these points.

It is clear that recolouring changing trails which start in points $Q_{i_{1}}, \ldots, Q_{i_{m}}$ establishes a bijection between $O_{0}$ and $O_{1}$.

On the other hand, each object in $O_{0}$ belongs to some $s_{\lambda^{\prime \prime} / \mu^{\prime \prime}} s_{\sigma^{\prime \prime} / \tau^{\prime \prime}}$ : Denote the set of all the corresponding quadruples $\left(\lambda^{\prime \prime}, \mu^{\prime \prime}, \sigma^{\prime \prime}, \tau^{\prime \prime}\right)$ which occur in this sense by $S_{0}$. The same consideration applies to $O_{1}$ : Denote by $S_{1}$ the corresponding set of quadruples $\left(\lambda^{\prime}, \mu^{\prime}, \sigma^{\prime}, \tau^{\prime}\right)$.

Lemma 15 Given the above definitions, we have the following "generic" identity for skew Schur functions:

$$
\sum_{\left(\lambda^{\prime}, \mu^{\prime}, \sigma^{\prime}, \tau^{\prime}\right) \in S_{1}} s_{\lambda^{\prime} / \mu^{\prime}} s_{\sigma^{\prime} / \tau^{\prime}}=\sum_{\left(\lambda^{\prime \prime}, \mu^{\prime \prime}, \sigma^{\prime \prime}, \tau^{\prime \prime}\right) \in S_{0}} s_{\lambda^{\prime \prime} / \mu^{\prime \prime}} s_{\sigma^{\prime \prime} / \tau^{\prime \prime}}
$$

This statement is certainly as general as useless: Let us specialize to a somewhat "friendlier" assertion.

Lemma 16 Given the above definitions, assume that all black points have the same parity, and that all white points have the same parity. Then (8) specializes to

$$
s_{\lambda / \mu} s_{\sigma / \tau}=\sum_{\left(\lambda^{\prime}, \mu^{\prime}, \sigma^{\prime}, \tau^{\prime}\right) \in S_{1}} s_{\lambda^{\prime} / \mu^{\prime}} s_{\sigma^{\prime} / \tau^{\prime}}
$$

where $S_{1}$ encompasses all the quadruples $\left(\lambda^{\prime}, \mu^{\prime}, \sigma^{\prime}, \tau^{\prime}\right)$ which correspond to any twocoloured graph object that can be obtained by recolouring the changing trails starting in points $Q_{i_{1}}, \ldots, Q_{i_{m}}$ in any "initial" two-coloured graph object from $s_{\lambda / \mu} s_{\sigma / \tau}$.

Proof: Without loss of generality we may assume that all even points are white and all odd points are black in $s_{\lambda / \mu} s_{\sigma / \tau}$. By recolouring changing trails, all the points $Q_{i_{1}}, \ldots, Q_{i_{m}}$ are matched with points of opposite colour and parity.

So if $Q_{i}$ is odd and black, then the recolouring trail starting at $Q_{i}$ connects it which some other point $Q_{k}$ which is even and white: After recolouring, $Q_{i}$ is odd and white, and the recolouring operation altogether yields some two-coloured graph object belonging to some $s_{\lambda^{\prime} / \mu^{\prime}} s_{\sigma^{\prime} / \tau^{\prime}}$.

Now if we apply the recolouring operation to an arbitrary object from $s_{\lambda^{\prime} / \mu^{\prime}} s_{\sigma^{\prime} / \tau^{\prime}}$, the only possible partners for "wrongly-coloured" $Q_{i}$ (odd, but white) is another "wronglycoloured" $Q_{j}$ (even, but black). Hence this operation takes objects from $s_{\lambda^{\prime} / \mu^{\prime}} s_{\sigma^{\prime} / \tau^{\prime}}$ back to $s_{\lambda / \mu} s_{\sigma / \tau}$. 


\section{Proof of the Plücker relations}

In order to show that the general assertions of Section 5 do in fact lead to some interesting identities, we give a proof of the Plücker relations (Theorem 5), which is based on Lemma 16.

Proof: In the notation of Section 5, let

$$
\lambda=\left(2 n(n-1), 2(n-1)^{2}, \ldots, 4(n-1), 2(n-1)\right)
$$

and

$$
\sigma=((2 n-1)(n-1),(2 n-3)(n-1), \ldots, 3(n-1), n-1),
$$

$\mu=\tau=(0, \ldots, 0)$; and choose horizontal offset $t=0$. I.e., interpret $s_{\lambda} s_{\sigma}$ as the generating function of two-coloured graph objects consisting of two $n$-tuples of nonintersecting lattice paths, coloured green and blue, respectively, where green path $P_{i}^{g}$ starts at $(-i, 1)$ and ends at $\left(\lambda_{i}-i, N\right)$, and where blue path $P_{i}^{b}$ starts at $(-i, 1)$ and ends at $\left(\sigma_{i}-i, N\right)$.

Observe that this setting obeys the assumptions of Lemma 16.

Now consider the set of green endpoints $\left\{Q_{1}, \ldots, Q_{k}\right\}$, where $Q_{i}=\left(\lambda_{r_{i}}-r_{i}, N\right)$. (Here, $1 \leq r_{1}<r_{2}<\cdots<r_{k} \leq n$ is the fixed list of integers from Theorem 5.) Recolouring changing trails which start at these points amounts to determining the set $\left\{R_{1}, \ldots, R_{k}\right\}$ of respective endpoints of the changing trails, and changing colours.

Assume that $R_{i}=\left(\sigma_{s_{i}}-s_{i}, N\right)$, then in terms of the associated Schur functions Lemma 16 directly leads to the identity:

$$
s_{\lambda} s_{\sigma}=\sum_{1 \leq t_{1}<\cdots<t_{k} \leq n} s_{\left(\lambda_{1}, \ldots, \sigma_{t_{1}}, \ldots, \sigma_{t_{k}}, \ldots, \lambda_{n}\right)} s_{\left(\sigma_{1}, \ldots, \lambda_{r_{1}}, \ldots, \lambda_{r_{k}}, \ldots, \sigma_{n}\right)},
$$

where the notation of the summands means that parts $\lambda_{r_{i}}$ were exchanged with parts $\sigma_{t_{i}}$, respectively. By the Jacobi-Trudi identity (4) and Observation 4, (5) and (10) are in fact equivalent.

Remark 17 In fact, even the quite general assertion of Lemma 15 can be generalized further: So far, our lattice paths always had starting points and end points at the same horizontal lines $(., 1)$ and $(., N)$, corresponding to the range of variables $x_{1}, \ldots, x_{N}$. Dropping this constraint yields Schur functions with different ranges of variables (e.g., $s_{\lambda}\left(x_{4}, x_{5}, x_{6}\right)$ ). Recalling that (see Remark 13) we actually also do allow partitions of different lengths, it is easy to see that Theorem 5 in [12] (which is a generalization of Ciucu's Schur function identity (6)) can be proved in the same way as Lemma 15.

\section{Kleber's Theorem}

The theorem [11, Thm. 3.2] is expressed in terms of certain operations on Ferrers boards (called Young diagrams in [11]): In order to state it, we need to describe the relevant notation. 
Figure 7: Illustration of outer corners and special drawing of Ferrers board for partition $\lambda=(8,6,5,3,3,1,1)$.

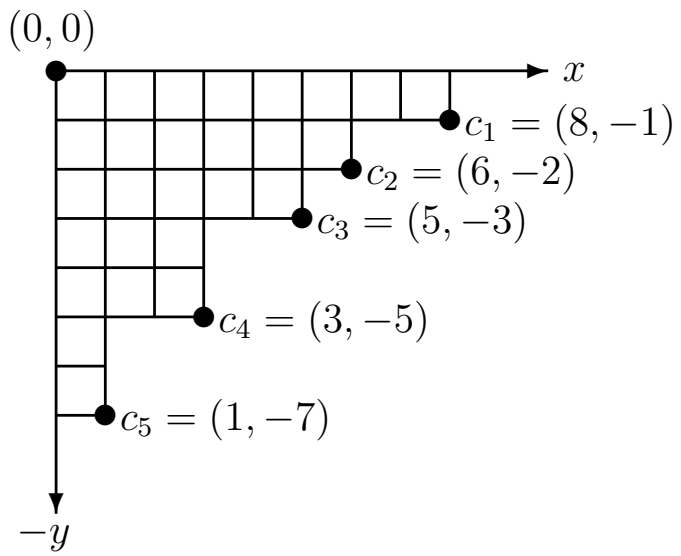

First, we introduce a particular way of drawing the Ferrers board of $\lambda=\left(\lambda_{1}, \ldots, \lambda_{r}\right)$ in the plane: Let $x_{1}>x_{2}>\cdots>x_{n}>x_{n+1}=0$ be the ordered list of the distinct parts contained in $\lambda$; set $y_{i}=$ the number of parts of $\lambda$ which are $\geq x_{i}$.

Setting $y_{0}=0$, we have $0=y_{0}<y_{1}<\cdots<y_{n}$, and $\left(x_{i}\right),\left(y_{i}\right)$ simply yield another encoding of the partition $\lambda$ :

$$
\lambda=\left(x_{1}^{y_{1}-y_{0}}, x_{2}^{y_{2}-y_{1}}, \ldots, x_{n}^{y_{n}-y_{n-1}}\right) .
$$

Now consider the $n$ points $\left(x_{1},-y_{1}\right),\left(x_{2},-y_{2}\right), \ldots,\left(x_{n},-y_{n}\right)$ in the plane: The Ferrers board of $\lambda$ is represented as the set of points $(x,-y)$ such that:

$$
\begin{aligned}
x \geq 0 & \text { and } y \geq 0, \\
x \leq x_{i} & \text { and } y \leq y_{i} \text { for some } i .
\end{aligned}
$$

Figure 7 illustrates this concept. The $n$ points $c_{1}=\left(x_{1},-y_{1}\right), c_{2}=\left(x_{2},-y_{2}\right), \ldots, c_{n}=$ $\left(x_{n},-y_{n}\right)$ are called outside corners, the $n+1$ points $\left(x_{1},-y_{0}\right),\left(x_{2},-y_{1}\right), \ldots,\left(x_{n+1},-y_{n}\right)$ are called inside corners.

Now we are in a position to define two operations on partitions: In the above notation, take two integers $i, j$ such that $1 \leq i \leq j \leq n$ and define two partitions derived from the original $\lambda$ via manipulating the inside and outside corners of its associated Ferrers board:

$$
\begin{aligned}
& \pi_{j}^{i}(\lambda): \text { add } 1 \text { to each of } x_{i+1}, \ldots, x_{j} ; y_{i}, \ldots, y_{j}, \\
& \mu_{j}^{i}(\lambda): \text { add }-1 \text { to each of } x_{i+1}, \ldots, x_{j} ; y_{i}, \ldots, y_{j} .
\end{aligned}
$$

These operations add or remove, respectively, a border strip that reaches from the $i$-th outside corner to the $j$-th inside corner (see Figure 8).

We need to add or remove nested border strips: Given integers $1 \leq i_{1}<\cdots<i_{k} \leq$ $j_{k}<\ldots j_{1} \leq n$, we define

$$
\begin{aligned}
\pi_{j_{1}, \ldots, j_{k}}^{i_{1}, \ldots, i_{k}} & =\pi_{j_{1}}^{i_{1}} \circ \cdots \circ \pi_{j_{k}}^{i_{k}}, \\
\mu_{j_{1}, \ldots, j_{k}}^{i_{1}, \ldots, i_{k}} & =\mu_{j_{1}}^{i_{1}} \circ \cdots \circ \mu_{j_{k}}^{i_{k}} .
\end{aligned}
$$


Figure 8: Illustration of operations $\pi_{j}^{i}$ and $\mu_{j}^{i}$ for $i=2, j=5$ applied to $\lambda=$ $(8,6,5,3,3,1,1)$.
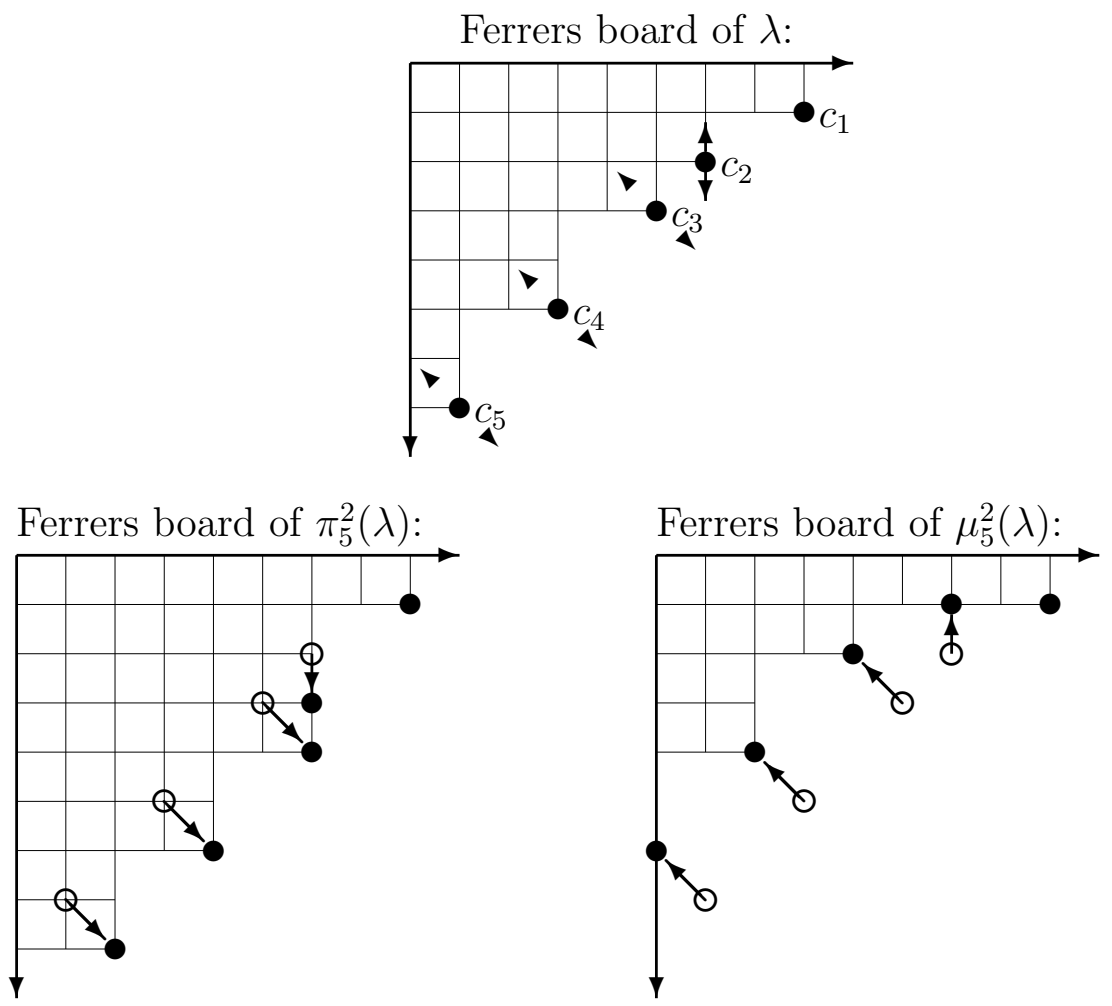
Figure 9: Illustration of operation $\lambda \pm \omega_{l}$ for $l=y_{4}=5$, applied to $\lambda=(8,6,5,3,3,1,1)$.
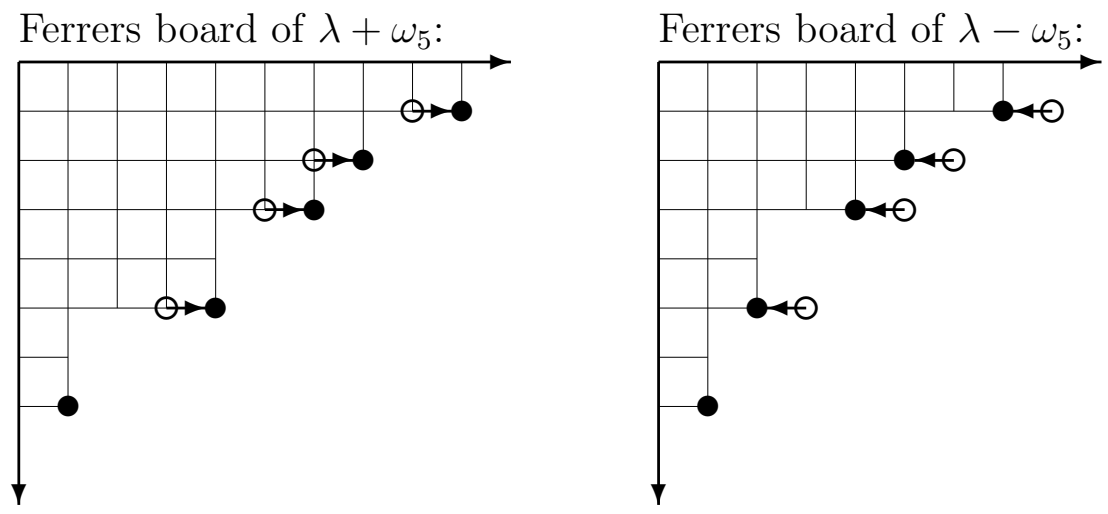

Note that the corners which are shifted by these operations might not appear as corners in the geometric sense any more; nevertheless we consider them as the object for subsequent operations $\pi$ and $\mu$ : Nesting $\pi$ and $\mu$ in this sense yields something which can be interpreted again as a partition, since we always have $x_{i} \geq x_{i+1}$ and $y_{i} \leq y_{i+1}$ (see Figure 8.)

The last operation we need is the following: In the above notation, let $k$ be an integer, $1 \leq k \leq n$. Clearly, the Ferrers board contains at least one column of length $l=y_{k}$ : Adding or removing some column of length $l$ amounts to adding \pm 1 to all coordinates $x_{i}$, $1 \leq i \leq k$. We denote this operation by $\lambda \pm \omega_{l}$. (See Figure 9.)

Theorem 18 (Theorem 3.2 in [11]) Let $\lambda=\left(\lambda_{1}, \lambda_{2}, \ldots, \lambda_{r}\right)$ be a partition with $n$ outside corners. For an arbitrary integer $k, 1 \leq k \leq n$, set $l=y_{k}$ (in the above notation). Then we have:

$$
s_{\lambda} s_{\lambda}=s_{\lambda+\omega_{l}} s_{\lambda-\omega_{l}}+\sum_{m \geq 1} \sum_{\substack{1 \leq i_{1}<\cdots<i_{m} \leq k \\ k \leq j_{m}<\cdots<j_{1} \leq n}}(-1)^{m-1} s_{\pi_{j_{1}, \ldots, j_{m}}^{i_{1}, \ldots, i_{m}}(\lambda)} s_{\mu_{j_{1}, \ldots, j_{m}}^{i_{1}, \ldots, i_{m}}(\lambda)} .
$$

The connections between Ferrers boards and nonintersecting lattice paths were illustrated in Section 3: Here we have to give the proper "translation" of operations $\pi_{j}^{i}$ and $\mu_{j}^{i}$ to nonintersecting lattice paths.

First observe that the outside corners of a partition correspond to blocks of consecutive endpoints (here, consecutive means "having distance 1 in the horizontal direction") in the lattice path interpretation: Number these blocks from right to left by $1,2, \ldots, n$, and denote the additional block of (consecutive) starting points by $n+1$ (see the upper picture in Figure 10).

Interpret some object from $s_{\lambda} s_{\lambda}$ in the same way as in Section 5. More precisely, let $\sigma=\lambda, \mu=\tau=0$ and horizontal offset $t=1$ in the general definitions preceding Lemma 15. Figure 10 illustrates the position of starting points and end points of the corresponding lattice paths: Blue points are drawn as black dots, green points are drawn as white dots; blocks are indicated by horizontal braces. 
Figure 10: Illustration of operations $\pi_{j}^{i}$ and $\mu_{j}^{i}$ for $i=2, j=4$ applied to $\lambda=$ $(8,6,5,3,3,1,1)$, translated to lattice paths.

Terminal points for $(\lambda, \lambda)$ :

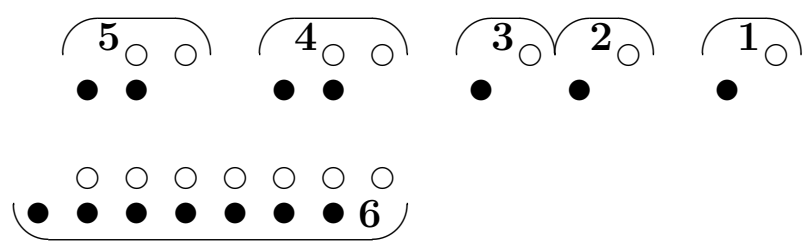

Terminal points for $\left(\pi_{5}^{2}(\lambda), \mu_{5}^{2}(\lambda)\right)$ :

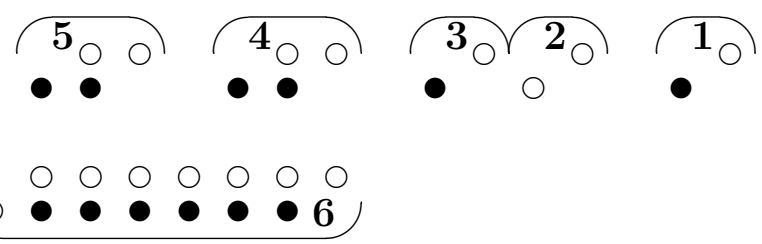

Figure 11: Illustration of operations $\lambda \pm \omega_{l}$ for $l=y_{4}=5$, applied to $\lambda=(8,6,5,3,3,1,1)$, translated to lattice paths.

Terminal points for $\left(\lambda+\omega_{5}, \lambda-\omega_{5}\right)$ :
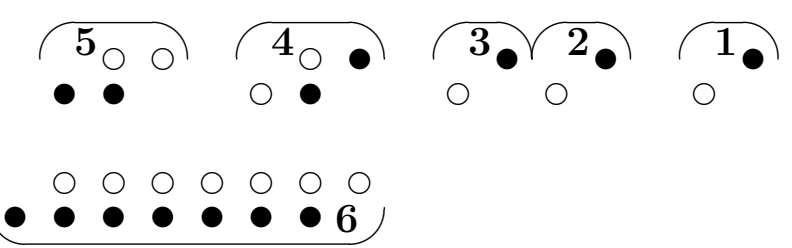

It is easy to see that the simultaneous application of $\pi_{j}^{i}$ to the "green object" and of $\mu_{j}^{i}$ to the "blue object" amounts to interchanging colours of the leftmost point in blue block $i$ and of the appropriate endpoint of a corresponding changing trail in block $j+1$ (i.e., the rightmost point in green block $j+1$ if $j<n$, or the the leftmost point in blue block $n+1$ if $j=n$; see Figure 10).

Likewise, adding some column of height $l=y_{k}$ to the "blue object" and simultaneously removing such column from the "green object" amounts to interchanging colours of the leftmost blue point and the rightmost green point in blocks $1,2, \ldots, k$ if $k<n$; if $k=n$, then the same effect can be achieved by interchanging colours of the leftmost blue point and the rightmost green point in block $n+1$. (See Figure 11.)

Proof of Theorem 18: Consider a two-coloured object from $s_{\lambda} s_{\lambda}$ in the lattice path interpretation. As in Section 5, we look at the noncrossing perfect matching that the changing 
trails induce among their $2 n+2$ endpoints, the leftmost and rightmost points in blocks $1, \ldots, n, n+1$. Note that in the case of $s_{\lambda} s_{\lambda}$, the parity constraint and the colour constraint of Lemma 14 coincide.

Now consider the $k$ changing trails which begin at the leftmost (blue) endpoints of blocks $1,2, \ldots, k$. There are exactly two cases:

1. The changing trails match these points up with the rightmost (green) endpoints of blocks $1,2, \ldots, k$ : Then recolouring these $k$ trails results in an object of type $s_{\lambda+\omega_{l}} s_{\lambda-\omega_{l}}$. Conversely, given an object of type $s_{\lambda+\omega_{l}} s_{\lambda-\omega_{l}}$, the parity and colour constraints of Lemma 14 force the points in blocks $1,2, \ldots, k$ to be matched amongst themselves, so they are in bijection with this subset of $s_{\lambda} s_{\lambda}$ objects.

2. Otherwise, some of those $k$ points must match up with points in blocks $k+1, \ldots, n+$ 1. Suppose there are $m$ such matchings, and that they match the leftmost points in blocks $i_{1}<i_{2}<\cdots<i_{m}$ with points in blocks $j_{1}>j_{2}>\cdots>j_{m}$. (Since the changing trails cannot cross, we in fact know that $i_{r}$ is matched with $j_{r}$, for $1 \leq r \leq m$.) Recolouring these $m$ trails gives an object of type $s_{\pi_{j_{1} \ldots j_{m}}^{i_{1} \ldots i_{m}}}(\lambda) s_{\mu_{j_{1} \ldots j_{m}}^{i_{1} \ldots i_{m}}}(\lambda)$. This time, though, we do not have a bijection. Given an object of

$$
s_{\pi_{j_{1} \ldots j_{m}}^{i_{1} \ldots i_{m}}}(\lambda) s_{\mu_{j_{1} \ldots j_{m}}^{i_{1} \ldots i_{m}}}(\lambda)
$$

the same parity and colour constraints of Lemma 14 do guarantee that $m$ changing trails connect each $i_{r}$ with $j_{r}$. However, when we recolour them to get an object of $s_{\lambda} s_{\lambda}$, we may arrive at an object that has other changing trails leaving blocks $1, \ldots, k$, aside from the $m$ we considered.

Thus, we are in the "typical" situation for an inclusion-exclusion argument, which immediately yields equation (11).

This finishes the proof.

Remark 19 When $k=1$, both cases of the above proof amount to recolouring the trail beginning at the rightmost green endpoint, so this is a special case of Lemma 16. The $k=n$ case follows similarly, after exchanging blue and green.

\section{References}

[1] D. Bressoud, Proofs and Confirmations: The Story of the Alternating Sign Matrix Conjecture, Cambridge University Press, New York, 1999.

[2] C. L. Dodgson, Condensation of Determinants, Proceedings of the Royal Society of London 15 (1866), 150-155.

[3] M. Fulmek, A Schur function identity, J. Combinatorial Theory A 77 No. 1 (1997). 
[4] M. Fulmek and C. Krattenthaler, Lattice path proofs for determinant formulas for symplectic and orthogonal characters, J. Combinatorial Theory A 77 (1997), $3-50$.

[5] W. Fulton and J. Harris, Representation Theory, Springer, New York, 1991.

[6] I. M. Gessel and X. Viennot, Determinants, paths, and plane partitions, preprint, 1988.

[7] I. P. Goulden, Quadratic Forms of Skew Schur Functions, European J. of Combinatorics, 9 (1988), 161-168

[8] A. Hamel, private communication, 1997.

[9] C.G.J. JacoBI, De formatione et proprietatibus Determinantium, in: Gesammelte Werke, vol. 3, Georg Reimer, Berlin (1884), 355-392; first published in Journal für Reine und Angewandte Mathematik 22 (1841), 285-318.

[10] A. N. KiRILlov, Completeness of states of the generalized Heisenberg magnet. (Russian), Zap. Nauchn. Sem. Leningrad. Otdel. Mat. Inst. Steklov. (LOMI) 134 (1984), transl. in J. Soviet Math. 36 (1987), 115-128

[11] M. KLeBer, Plücker Relations on Schur Functions, Journal of Algebraic Combinatorics, to appear.

[12] C. Krattenthaler, Schur Function identities and the number of perfect matchings of holey Aztec rectangles, in: " $q$-series from a Contemporary Perspective", M.E.H. Ismail, D. Stanton, eds., Contemporary Math. 254, Amer. Math. Soc., Providence, R.I., 2000, 335-350.

[13] I. G. Macdonald, Symmetric Functions and Hall Polynomials, Oxford University Press, New York/London, 1979.

[14] T. Muir, The theory of determinants in the historical order of development, 4 vols., Macmillan, London, 1906-1923.

[15] J. Propp and R. P. Stanley, Domino tilings with barriers, J. Combinatorial Theory (A) 87 (1999), 347-356.

[16] B. E. Sagan, The symmetric group, Wadsworth \& Brooks/Cole, 1991.

[17] R. P. Stanley, Theory and applications of plane partitions: Part 1,2, Stud. Appl. Math 50 (1971), 167-188, 259-279.

[18] R. P. Stanley, Enumerative Combinatorics, Vol. 2, Cambridge Studies in Advanced Mathematics 62, Cambridge University Press, 1999.

[19] D. Stanton and D. White, Constructive Combinatorics, Undergraduate Texts in Math., Springer-Verlag New York, Berlin, Heidelberg, Tokyo, 1986. 
[20] J. R. Stembridge, Nonintersecting paths, pfaffians and plane partitions, Adv. Math. 83 (1990), 96-131.

[21] B. Sturmfels, Algorithms in Invariant Theory, Texts and Monographs in Symbolic Computation, Springer-Verlag, Wien, (1993).

[22] H.W. Turnbull, The Theory of Determinants, Matrices, and Invariants, Dover Publications, New York, (1960).

[23] D. Zeilberger, Dodgon's Determinant-Evaluation Rule proved by TWO-TIMING MEN and WOMEN, Elect. J. of Combinatorics 4(2) [Wilf Festschrift volume], 1997.

[24] D. Zeilberger, A combinatorial approach to matrix algebra, Discrete Math. 56, (1985), 61-72. 